\title{
Homeotic genes controlling flower development in Antirrhinum
}

\author{
ENRICO S. COEN, SANDRA DOYLE, JOSE M. ROMERO*, ROBERT ELLIOTT, RUTH MAGRATH \\ and ROSEMARY CARPENTER \\ John Innes Institute, AFRC Institute of Plant Science Research, Colney Lane, Norwich NR4 7UH, UK \\ * Permanent address: Instituto de Bioquimica Vegetal y Fotosintesis, Universidad de Sevilla, CSIC Apdo. 1113, 41080-Sevilla, Spain
}

\section{Summary}

In order to study genes controlling flower development, we have carried out an extensive transposon-mutagenesis experiment in Antirrhinum majus. More than 15 independent homeotic mutations were obtained, allowing three categories of genes to be defined. The first includes floricaula $(f l o)$, a primary gene required for the initiation of the floral developmental pathway. In the absence of the wild-type flo product, proliferating inflorescence meristems arise in place of flowers. The flo gene has been isolated and shown to be expressed transiently in a subset of organ primordia in the floral meristem. The second category includes genes that affect the identity, and also sometimes the number, of whorls of organs in the flower. These genes act in overlapping domains so that each whorl has a distinct combination of gene functions, suggesting a model for the genetic control of whorl identity and number. Genes of the third category control differences between organs in the same whorl and hence the overall symmetry of the flower. We discuss how the basic plan of the flower and inflorescence may arise through the interactions between the three categories of genes.

Key words: homeotic genes, Antirrhinum, floral development, transposon-mutagenesis.

\section{Introduction}

Most of our current notions of how homeotic genes act come from studies on segmentation in Drosophila (see articles by Nusslein-Volhard et al. and Garber et al., in this volume). In certain respects there are many similarities between homeotic genes in plants and those in Drosophila. In both cases, the genes switch developmental pathways of homologous structures and many of the genes appear to interact in a combinatorial manner. However, there are also some important differences that reflect the distinct modes of growth. One of these is that organs are produced throughout a plant's life history from meristems by sequential addition, giving the potential for indeterminate growth. The segments of Drosophila are produced synchronously by subdivision of an egg of finite size. The additive and sequential plant growth pattern is reflected in the homeotic phenotypes which can result in premature developmental arrest or continuous reiteration of a developmental programme. Consequently, as well as being homeotic, some of the mutations can be considered to be heterochronic because they change the relative timing of developmental events.

Perhaps one of the great advantages of plants is that many different species have been studied and characterised genetically: maize, barley, wheat, tomato, pea, Arabidopsis, Antirrhinum (King, 1974). This provides a very valuable resource for comparative studies of gene action. At present, the molecular and genetic analysis of floral homeotic genes is most advanced in two species: Antirrhinum majus and Arabidopsis thaliana. Antirrhinum has several advantages: well characterised transposable elements, large flowers which are easy to emasculate and cross and ease of vegetative propagation. Arabidopsis has different merits: small genome size, rapid generation time, ease of transformation and each plant occupies relatively little space. This paper and that of Schwarz-Sommer et al. in this volume present some recent findings in studies on Antirrhinum. Studies on Arabidopsis are presented by Meyerowitz et al. in this volume.

\section{Results and discussion}

Description of wild type and mutagenesis strategy Following germination of seed, the initial growth of a wild-type Antirrhinum plant above ground level is generated from a vegetative apical meristem which usually produces a pair of opposite $\left(180^{\circ}\right)$ leaves at each node in a decussate phyllotaxis (i.e. each pair is at a right angle to the previous one). After a period of vegetative growth, the apex undergoes a transition to become an inflorescence meristem (Fig. 1A). In comparison with vegetative growth, the inflorescence 
meristem produces much smaller leaves (bracts) in a spiral arrangement, generally with a single bract at each node separated by short internodes. In the axils of these bracts, floral meristems are initiated which produce four concentric whorls of organs separated by very short 'internodes' so that consecutive whorls appear adjacent to each other. The parts of a plant may therefore be considered as being generated by three homologous meristems: vegetative, inflorescence and floral, which differ in their respective phyllotaxis (decussate, spiral, whorled), organs produced (leaves, bracts, floral organs) and internode length (long, short, very short).

Antirrhinum flowers are zygomorphic: they can be divided into two halves by a single longitudinal plane which passes through the vertical axis (Fig. $1 B$ and $C$ ). The flower consists of four whorls of organs, numbered starting from the outside of the flower; thus whorl 1 is outermost and whorl 4 is central (Fig. 1B). The whorl primordia appear sequentially: whorl 1 first, then whorls 2 and 3 almost simultaneously and finally whorl 4 (Awasthi et al. 1984). Organs within a whorl are referred to as upper or lower, depending on their position relative to the bract and stem (bract is lower, stem is upper, Fig. 1B). Whorl 1 comprises five sepals, the lowest two being alternate to the bract. The corolla occupies whorl 2 and consists of five petals, alternate with the sepals. The petals are united for part of their length to form a tube which terminates in five lobes (Fig. 1C). The two upper lobes have a very distinct shape from the lower three and the junction between the upper and lower lobes forms a hinge region which allows the flower to be opened by insects. In whorl 3, five stamen primordia are initiated alternately with the petals but the uppermost primordium fails to develop fully and yields an aborted or rudimentary structure. Consequently, the adult flower has only four stamens, the upper two being shorter than the lower pair. Whorl 4 is occupied by two united carpels forming a gynoecium with a bilocular ovary.

In order to isolate and study homeotic genes in Antirrhinum we have carried out an extensive transposon mutagenesis experiment (Carpenter and Coen, 1990 ). Plants carrying active transposons were grown at $15^{\circ} \mathrm{C}$, a temperature which generally increases transposition rates in Antirrhinum (Carpenter et al. 1987; Harrison and Fincham, 1964), and self-pollinated to give 26000 progeny, many of which were expected to carry recessive mutations in a heterozygous condition. The homozygous phenotypes were revealed by selfpollination of these plants to give a population of 80000 plants. Over 15 independent homeotic mutations were obtained from this screen and here we describe the results and conclusions derived from the analysis of some of the genes involved.

\section{Genetic control of first steps in floral development}

The transition from inflorescence to floral meristems represents the first step specific to the floral developmental pathway (Fig. 1A). Mutants that are unable to carry out the transition between inflorescence and floral meristems might be expected to produce proliferating inflorescence shoots in place of flowers. One mutant obtained from the transposon mutagenesis experiment showing this phenotype is floricaula $(f l o)$ (Carpenter and Coen, 1990). The flo mutant initiates vegetative growth and the transition to inflorescence meristem in a similar manner to wild type. Instead of flowers being produced in the axils of bracts, indeterminate shoots bearing further bracts are produced, each shoot having two opposite bracts at the base followed by a spiral of single bracts. Each of these shoots can in turn produce further shoots in the axils of their bracts and this sequence can repeat itself indefinitely. The wild-type flo product is therefore necessary for the transition between inflorescence and floral meristems so that in its absence the inflorescence programme is continually reiterated (Fig. 2). The flo mutant can be considered to be homeotic because it results in one structure (the flower) being replaced by a homologous structure (indeterminate shoot). The mutant can also be viewed as heterochronic (changing the relative timing of developmental events) because it results in an early developmental programme (inflorescence meristem) being continually reiterated.

Other mutants have been described in Antirrhinum, such as squamosa and squamata, which produce inflorescence-like shoots in the axils of bracts, although many of these shoots eventually produce flowers (Stubbe, 1966; Chittenden, 1928; Schwarz-Sommer et al. 1990). It is not clear if the production of flowers reflects leakiness in the particular alleles of these genes or a specific property of the genes themselves.

A second class of mutant has almost the opposite effect of those described above because it promotes the conversion of inflorescence to floral meristems in positions where this would not normally occur. Plants with indeterminate inflorescences, such as Antirrhinum, do not produce terminal flowers (see below) Thus, the apical inflorescence meristem does not itself undergo the transition to a floral meristem. The centroradialis mutant in Antirrhinum produces terminal flowers, suggesting that the wild-type product of this gene prevents the conversion of the inflorescence apex to a floral meristem (Fig. 2; and Kuckuck and Schick, 1930). So far we have not observed a mutation with this phenotype in our mutagenesis experiment.

The flo gene of Antirrhinum has been isolated and extensively characterised (Coen et al. 1990). A plant obtained from the mutagenesis experiment was homozygous for an allele called flo-613 and initially needed to be propagated vegetatively by cuttings because of its lack of flowers. However, occasional flowers were observed on the propagated flo-613 mutant plants (Fig. 3) and seed of these flowers sometimes gave wildtype progeny, showing that flo-613 was genetically unstable and could sometimes revert to a wild-type allele. It was shown that reversion to wild type correlated with excision of a particular copy of the transposon Tam 3 in the genome, indicating that this copy was responsible for the mutant phenotype (Coen et al. 1990). The sequences flanking this copy were 


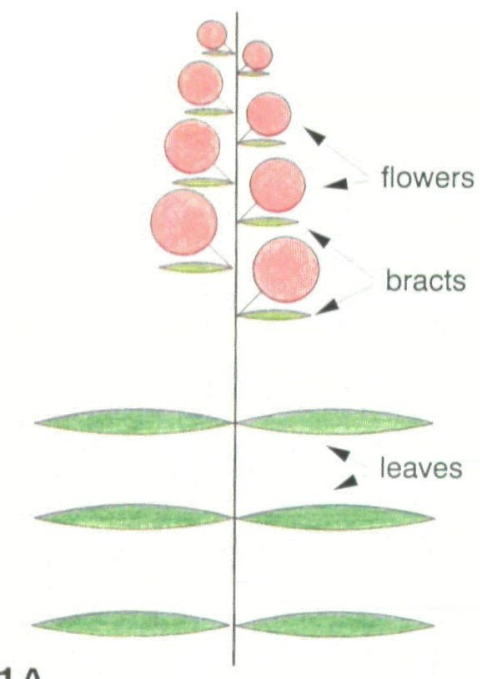

$1 \mathrm{~A}$
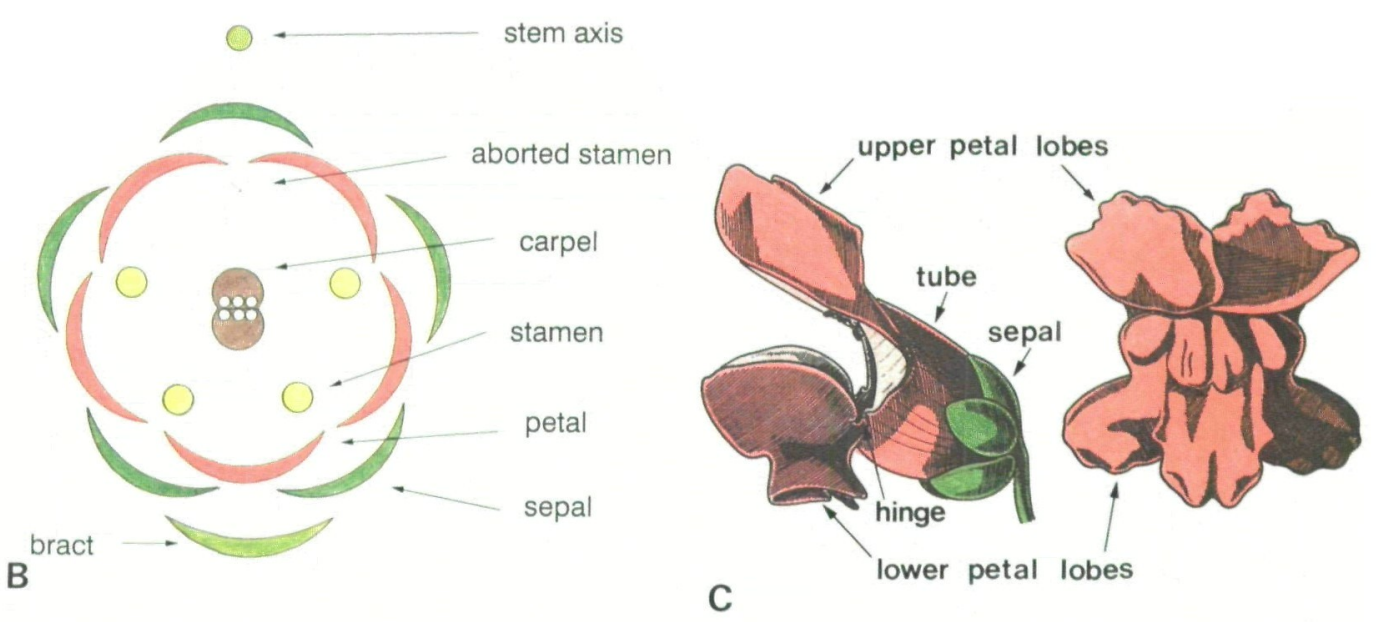

Fig. 1. Wild-type Antirrhinum. (A) Schematic illustration of plant. Lateral shoots arising in the axils of leaves are omitted for clarity. (B) Floral diagram. (C) Drawing of flower in side view (left) and face view (right). The flower in side view is shown slightly open to illustrate the hinge more clearly. Adapted from Weberling (1989).

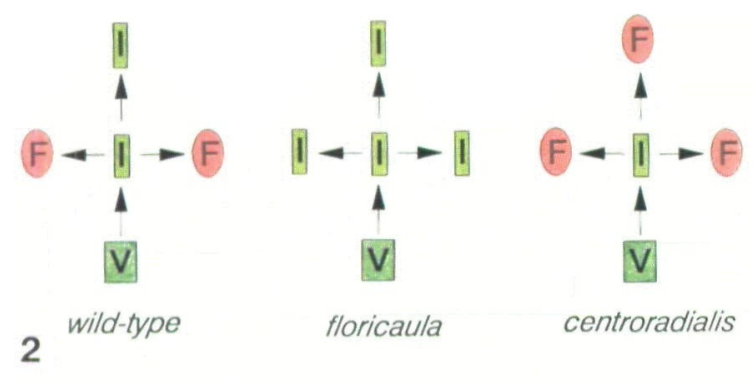

Fig. 2. Types of meristems in wild type, floricaula and centroradialis mutants. Abbreviations for meristems are $\mathrm{V}$, vegetative; $\mathrm{I}$, inflorescence; $\mathrm{F}$, floral.

Fig. 3. Unstable flo-613 mutant showing wild-type sectors. Filled arrows point to a wild-type flower and a wild-type secondary (axillary) inflorescence. Open arrows point axillary inflorescence shoots that are mutant and lack flowers.

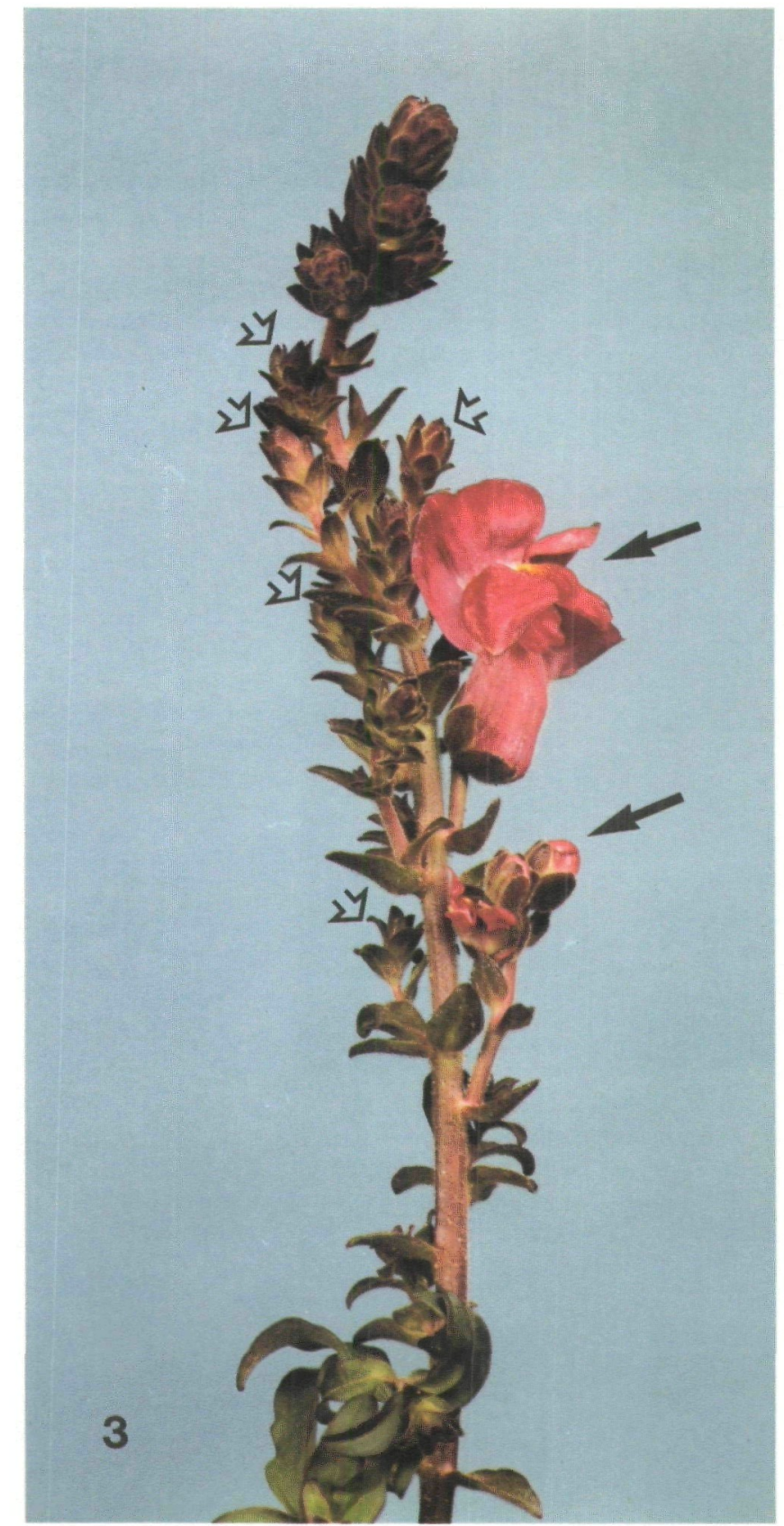



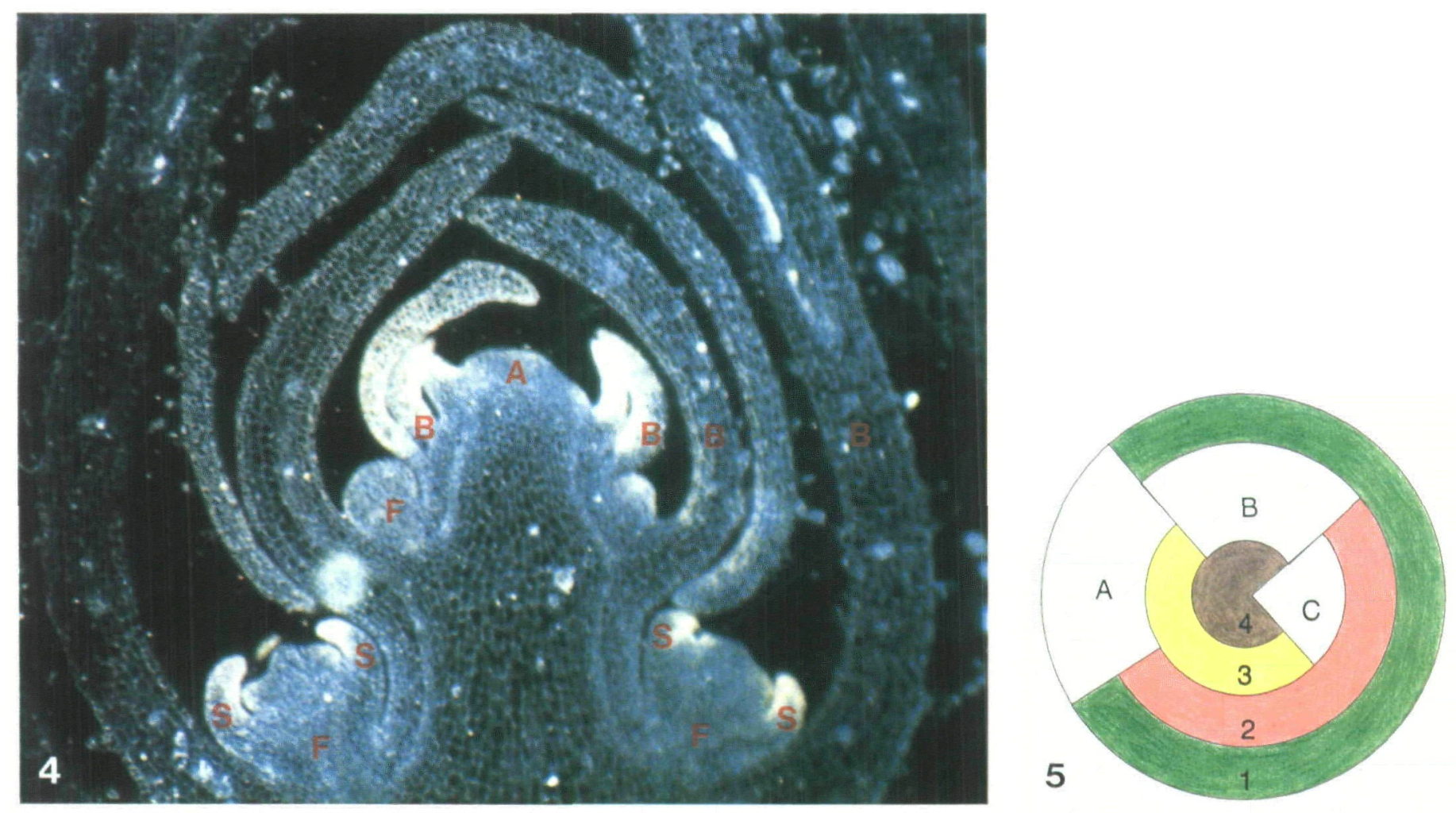

Fig. 4. In situ hybridisation of flo to a wild-type inflorescence, viewed under dark field. Regions are: A, inflorescence apex; B, bract primordia; F, floral meristems; S, sepal primordia. The primordıa at earlier developmental stages are near the inflorescence apex and progressively later stages are seen towards the bottom of the inflorescence. Signal is most clearly seen as a cream colour in the young bracts and sepal primordia. Only the upper part of the inflorescence is shown. The probe was labelled with digoxygenin (see Coen $e t$ al. 1990 , for details).

Fig. 5. Schematic illustration of the 4 whorls $(1-4)$ and 3 regions $(A, B, C)$ of a flower.

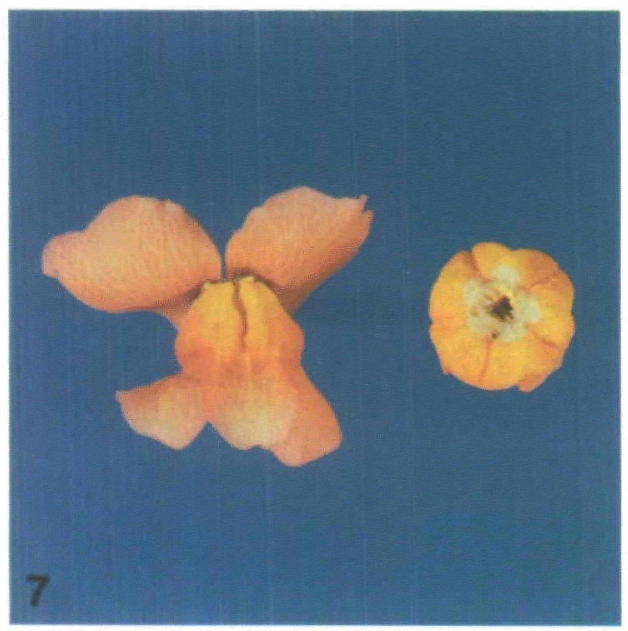

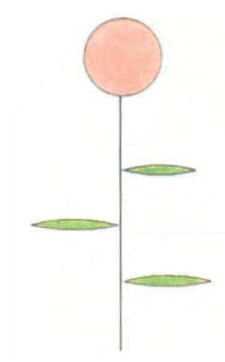

single terminal

9 flower

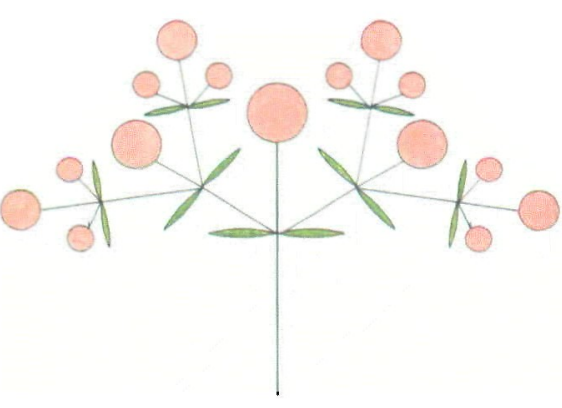

determınate

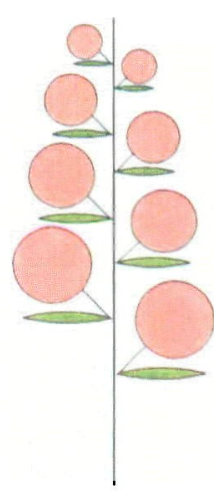

Indeterminate

Fig. 7. Flowers of wild-type (left) and an extreme $c y c$ mutant (right). The flowers are orange because they also carry several mutations (eos and sulf) in pigmentation genes.

Fig. 9. Examples of three different types of inflorescence. The particular determinate inflorescence shown is a dichasium and the indeterminate inflorescence is a raceme (Weberling, 1989). 
cloned using Tam 3 as a probe, allowing isolation of the flo locus.

The flo gene produces a transcript of about $1.6 \mathrm{~kb}$ which has the potential to encode a protein, FLO, of 396 amino acids. The FLO amino acid sequence shows no extensive homologies with other sequences in available data-banks (Coen et al. 1990). However, the protein contains a proline-rich $\mathrm{N}$ terminus and an acidic region; both of these features have been found in activation domains of transcription factors (Mermod $e t$ al. 1989; Struhl, 1989; Latchman, 1990). This suggests that the flo product may be a transcriptional activator, although other roles cannot be excluded. In situ hybridisation shows that $f l o$ is expressed from a very early stage in wild-type inflorescences in a very specific temporal and spatial sequence. The earliest expression seen is in bract primordia and is followed by expression in sepal, petal and carpel primordia, but no expression is seen in stamen primordia (Fig. 4). Expression in each organ is transient and is not observed in later stages of development (Coen et al. 1990).

Taken together, these results suggest that flo acts not only as a switch between inflorescence and floral meristems but is also involved in directing specific patterns of gene expression in the early fioral meristem. The expression of flo in certain primordia may be required to activate genes required for their normal development. Similarly, the absence of $f$ la from whorl 3 may be needed for normal stamen development. One important class of genes that may interact with flo in particular whorls are homeotic genes controlling whorl identity, and a possible model for such interactions will be discussed in detail below. However, it is important to emphasise that the expression of flo in a primordium need not imply a role for its development. For example, even though flo is expressed in bracts, it is not required for bract development because these organs apparently develop normally in flo mutants.

\section{Cell autonomy of flo}

As has already been mentioned, the flo-613 mutant occasionally produces wild-type flowers and sometimes whole racemes on an otherwise mutant plant (Fig. 3). In most cases, seed derived from these flowers gives mainly mutant progeny but sometimes the progeny segregate in the ratio 3 wild type: 1 mutant. The cases of germinal transmission of the revertant phenotype suggested that excision of Tam 3 from flo-613 occurred early in somatic development to give a heterozygous flower that was $\mathrm{Flo}^{+} / \mathrm{flo}-613$. However, the failure of germinal transmission in most cases shows that functional gametes can also be produced from homozygous mutant tissue (flo-613/flo-613). One explanation for this is that FLO is not essential for floral development and other FLO-independent pathways exist, allowing occasional flowers to be produced. This explanation does not seem to be correct because the flo- 640 allele, which arose as an imprecise Tam 3 excision from flo-613, has never been observed to produce flowers (Coen et al. 1990). A more probable explanation is that flo does not act in a cell-autonomous manner in the tissue giving rise to gametes. The lineage of cells giving rise to gametes is usually restricted to a particular cell layer in the flower (the LII) so that only events represented in this layer will be transmitted to progeny (Imai, 1934; TilneyBassett, 1986). It is possible that flo can act in the epidermal layer (LI) to direct floral development, so that Tam3 somatic excisions which restore gene function to the epidermis may allow development of a normal flower even though the genotype of its cells in the LII layer are still mutant.

\section{Genetic control of organ identity}

Other types of homeotic mutations obtained from transposon mutagenesis in Antirrhinum act after the transition from inflorescence to floral meristems because flowers are produced but the identity of whorls of organs in the flower are altered. Many of the genes involved affect the identities of organs in two adjacent whorls (Carpenter and Coen, 1990; Sommer et al. 1990; Schwartz-Sommer et al. this volume) and for convenience, three overlapping regions will be defined for wild type: A (whorls 1 and 2); B (whorls 2 and 3); C (whorls 3 and 4) (Fig. 5). One class of mutants, ovulata, affects region $\mathrm{A}$ and gives carpels instead of sepals in whorl 1 and stamens in place of petals in whorl 2, giving the overall phenotype carpel, stamen, stamen, carpel (Table 1). A second class affects region B and gives sepals instead of petals in whorl 2 and carpels instead of stamens in whorl 3, giving the phenotype sepal, sepal, carpel, carpel (whorl 4 does not always develop in these mutants). This class includes the deficiens (def), globosa ( $g l o$ ) and some sepaloidea (sep) mutants. A third class (e.g. pleni) affects region $\mathrm{C}$ and gives petals instead of stamens in whorl 3 and variable structures in whorl 4. Usually the latter mutations also affect the number of whorls and give extra whorls of petaloid organs within whorl 4. Because all of these classes of genes affect the

Table 1. Phenotypes of some whorl identity mutants in Antirrhinum

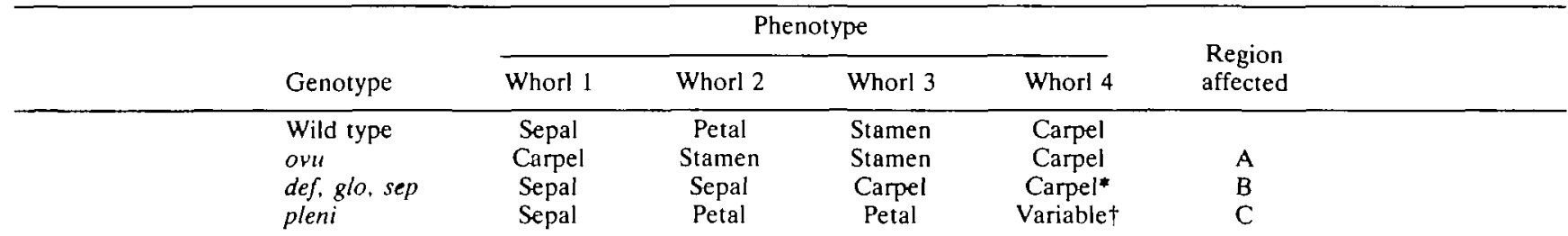

* Whorl 4 does not always develop in some of these mutants.

† Whorl 4 can be petaloid, sepaloid, carpelord or a mixture of these and extra petaloid whorls are produced within whorl 4. 
identity of whorls of organs they will be referred to as whorl identity genes (note that they do not affect the numbering of the whorls but only the identity of organs in the whorls). Similar classes of genes are observed in Arabidopsis (Bowman et al. 1989; Haughn and Sommerville, 1988; see also Meyerowitz et al. this volume), suggesting that the mechanisms controlling whorl identity have been highly conserved in evolution. It remains to be directly demonstrated, however, that these similarities reflect the action of homologous genes in the two species.

The action of many gene functions in overlapping regions could give each whorl a unique combination of functions. For example, if genes acting in regions $\mathrm{A}, \mathrm{B}$ and $\mathrm{C}$ are required for three functions $a, b$, and $c$ respectively, then the combination of functions in the four whorls of wild type would be: $a, a b, b c, c$. In principle, this might provide sufficient information to specify the identity of organs in each whorl. An important constraint on such a combinatorial model is that it needs to account for the particular mutant phenotypes observed when $a, b$ or $c$ are mutated. By this criterion, the simple model that the domains of $a, b$ and $c$ expression are established independently of each other does not fit the data. For example, if $a$ is required for sepal and petal development in region $\mathrm{A}$, how can these organ types develop outside this region in certain mutants (e.g. mutants lacking $c$ have petals in whorl 3 , see above)?

Consistent models can be constructed assuming that only two functions (e.g. $a$ and $b$ ) are established independently of each other (Carpenter and Coen, 1990). If only the interaction of $a$ and $b$ are considered, the combination of functions in the four wild-type whorls would be: $a, a b, b, 0$. Mutants in which $a$ is absent would give $0, b, b, 0$, consistent with the observed phenotype carpel, stamen, stamen, carpel. Mutants lacking $b$ would give $a, a, 0,0$, consistent with the observed phenotype sepal, sepal, carpel, carpel.

To incorporate the third function, $c$, in such a model, it is necessary to postulate that two of the functions ( $a$ and $c$ ) are not established independently of each other. For example, it has been proposed that $c$ might inhibit $a$ in region $\mathrm{C}$; thus a mutant lacking $c$ should be $a, a b, a b$, $a$, giving the phenotype: sepal, petal, petal, sepal (Carpenter and Coen, 1990). The first four whorls of $a g$ mutants of Arabidopsis have this phenotype (Yanofsky et al. 1990). The pleni mutants in Antirrhinum are also similar, although the fate of whorl 4 is variable in these mutants and can be sepaloid, petaloid, carpeloid or a mixture of these. This may reflect differences in the action of ag and pleni or differences in the particular alleles of these genes studied so far. A similar type of combinatorial model for the control of organ identity has also been proposed for Arabidopsis although in this case it has been suggested that $a$ inhibits $c$ in addition to $c$ inhibiting $a$ (see Meyerowitz et al. this volume).

\section{Regulation of whorl identity genes}

Two general types of model might be imagined for explaining the expression pattern of whorl identity genes. According to a purely spatial model, concentric fields could be set up in the early floral meristem, independently of the sequence of primordium initiation. The $a, b$ and $c$ functions could be activated in the appropriate field and hence specify the fate of primordia growing out from the various regions of the meristem. This type of model is formally similar to models proposed for the control of segment identity in Drosophila (Ingham, 1988). In the sequential type of model, which shares some features of early models of flower development (Heslop-Harrison, 1964), the consecutive growth of the primordia would be essential for the establishment of the domains of activity. The $a$, $b$ and $c$ functions would be activated in a manner that reflected the sequence of primordium initiation.

Some supporting evidence for a sequential model has come from studies on the flo gene of Antirrhinum. As described above, flo is expressed sequentially and appears to be activated in regions where primordia are being initiated. A further feature of flo expression is that it occurs transiently in bract, sepal, petal and carpel but not stamen primordia. This pattern of expression suggests that the flo gene acts not only as a switch between inflorescence and floral meristems but is also involved in directing specific patterns of gene expression in early floral meristems. In particular, it has been proposed that flo interacts in a sequential manner with the $a, b$ and $c$ whorl identity functions (Coen $e t a l$. 1990).

According to one model, as flo expression progresses from initiating bract to sepal and then petal primordia it activates $a$ in whorls 1 and 2, perhaps in combination with other gene products specific to the floral meristem (stages (i)-(iii), Fig. 6). Before flo expression has been established in whorl 3 , the $b$ and $c$ functions could be activated so that they become established in the presumptive whorl 3 cells. The combination of $b$ and $c$ (or $b$ in the absence of $a$ ) could then inhibit $f o$ expression and prevent flo from being established in the third whorl (stage (iii), Fig. 6). The factors activating $b$ and $c$ are unknown but may depend on a separate cascade of events initiated by early flo activity. Finally, $b$ is inactivated in whorl 4 , allowing expression of flo but because $c$ is already pre-established in this whorl, flo is unable to activate $a$ (stage (iv))

This model presents one plausible set of interactions, consistent with the data so far, although many other possibilities could be imagined. The model illustrates two important features of sequential systems. First, the fate of a whorl may depend on the temporal sequence in which gene expression is activated so that preestablishment of a gene function in one region changes its response when other genes are activated later. For example, in region $\mathrm{A}, a$ is activated early but in region $\mathrm{C}, c$ is established early and prevents activation of $a$. Second, early and late genes need not interact in a simple hierarchical fashion. For example, even though flo is activated earliest in the floral meristem as a whole, in cells giving rise to whorl 3 the $b$ and $c$ functions could be established earlier than flo and therefore regulate its expression. 
(l)

(Ii)

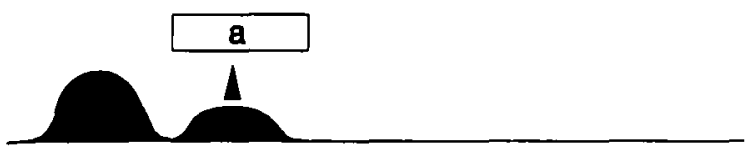

(iii)

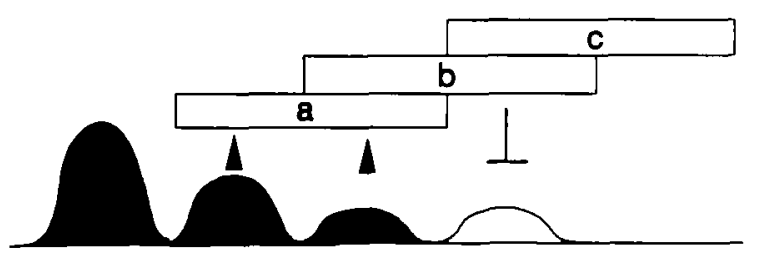

(iv)

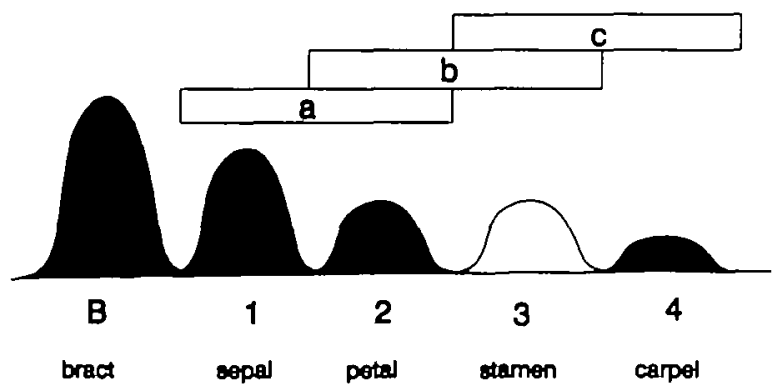

Fig. 6. Model for the interaction of flo with the whorl identity functions $a, b, c$. (i)-(iv) show progressive stages of the development of bracts and whorls 1-4. The developmental stage of each primordium is indicated by a dome which increases in height with time. It is important to emphasise that each dome does not represent the physical appearance of the primordium but only its developmental stage. It is likely that some of the small domes represent early stages of development which occur before the physical appearance of the primordia. The petal and stamen primordia are shown to be a similar stage because they appear almost simultaneously in development. Filled domes indicate flo expression, whereas open domes show no flo expression. The regions representing the $\mathrm{a}, \mathrm{b}$, c functions are illustrated by open boxes. In (ii) and (iii) arrows indicate possible activation of $a$ by flo and ( ) indicates inhibition of $f l o$. See text for full explanation.

\section{Genetic control of whorl number}

As described above, some of the whorl identity mutants also affect whorl number. Extreme mutants in some genes required for $b$ give fewer whorls than wild type. Mutants in genes needed for the $c$ function generally give an increase in whorl number and a more or less indeterminate growth pattern. The findings indicate that whorl identity genes can have two roles: the control of organ fate and the control of whorl number. The degree to which these separate roles are mediated by independent target genes is not fully understood. In all the mutants described above, determinate flowers have carpels in the final whorl whereas indeterminate flowers do not produce carpels. For example, although some def mutants in Antirrhinum have only three whorls, the third whorl contains carpels so the flower still has carpels in the final (central) whorl. However, the simplistic view that organ identity (i.e. production of a carpel) itself controls whorl number does not appear to be true because $o v u$ mutants have carpels in whorl 1 but still produce 4 whorls. It therefore seems likely that the control of whorl number and identity reflect activations of functions which are at least partially separable.

As described in the introduction, the flower can be considered as homologous to a shoot with an imposed determinate growth pattern. Support for this notion comes from the flo mutant phenotype which has indeterminate shoots in place of flowers. According to this view, the control of whorl number could be restated as the regulation of a determinacy or 'stop' function. Thus in wild-type flowers, determinacy is activated after four whorls are produced. In some of the mutants with fewer whorls than wild type, determinacy could be activated prematurely. In flowers with more than four whorls there is a delay or failure in the activation of determinacy.

Genes affecting both identity and number of homologous parts (i.e. segments) have also been described in Drosophila. However, unlike the floral mutants, the Drosophila mutants never give an increase in the number of homologous parts but only a reduction in number (Ingham, 1988). This may reflect a fundamental difference in the mechanism by which repetition of parts occurs in these two systems. In Drosophila, segmentation proceeds from an undivided structure of finite size, the egg, to a subdivided one, so that mutants failing to subdivide correctly give fewer segments. In flowers, whorl primordia are produced by sequential addition rather than by subdivision, giving the potential for indeterminate growth.

\section{Genetic control of differences between organs in the same whorl}

In radially symmetrical flowers (actinomorphic), all organs in the same whorl have very similar or identical morphologies. In zygomorphic flowers (e.g. Antirrhinum), one or more whorls contain organs with distinct morphologies. Examples of zygomorphic flowers include orchids, labiates (e.g. mints) and personates (e.g. Antirrhinum, Digitalis), the most striking feature of which are the very distinct upper and lower petals. Many mutations have been described in zygomorphic species that can reduce or eliminate the differences between organs in a whorl and thus render the flower more or less actinomorphic. These are usually homeotic because all the organs in a mutant whorl resemble one particular organ (generally the upper or lower organ) of the corresponding wild-type whorl.

Several different cycloidea (cyc) mutations have been described in Antirrhinum which give flowers with a more symmetrical appearance than wild type (Stubbe, 1966) and four such mutations were obtained from our transposon mutagenesis. Extreme cyc mutations give radially symmetrical flowers with all petals resembling the lowest petal of wild type (Fig. 7). Unlike wild type, in which the uppermost stamen is aborted, all five stamen primordia develop fully in these mutants to give mature organs of similar length to the lower stamens of wild type. These $c y c$ mutants also affect whorl 4 and 
give it a more symmetrical appearance than wild type. Thus, all organs in whorls 2, 3 and probably 4 resemble the lowest organs of the corresponding whorl in wildtype flowers. There are no major distinguishing features between upper and lower sepals in Antirrhinum so that it is not possible to assess the effects of $c y c$ alleles in whorl 1. However, whorl 1 is zygomorphic in some other species of the tribe Antirrhineae, suggesting that genes conferring zygomorphy (e.g. $C y c^{+}$) can act in whorl 1. Furthermore, analysis of double mutants of $c y c$ and $o v u$ in Antirrhinum indicates that cyc can affect whorl 1 (R. Carpenter and E. Coen, unpublished results).

In addition to the phenotype described above, there are also many cyc mutations which confer intermediate phenotypes. The various $c v c$ alleles can therefore be arranged as a series going from those that give fully symmetrical flowers to those conferring an almost wildtype phenotype. These alleles can be divided into two classes based on genetic complementation tests (Carpenter and Coen, 1990). Alleles from the same class fail to complement each other, whereas alleles from different classes show partial complementation giving almost wild-type $F_{1}$ hybrids with two small notches on the lower corolla lip. The $\mathrm{F}_{2}$ from the notched hybrids gives $50 \%$ notched and $25 \%$ of each parental type, indicating linkage between the alleles from the two classes. This suggests that cyc may be a complex locus composed of two interacting functional units. Alternatively, it is possible that there are two distinct $c y c$ genes which are tightly linked on the same chromosome and whose products interact.

As described earlier, many genes affect the identities of whorls, so it is possible to ask if the action of $c y c$ on particular organs depends on the whorl they occupy. For example, abortion of the uppermost organ in whor] 3 of wild type depends on the action of $c y c$ because in extreme cyc mutants all five stamen primordia develop fully. If stamens grow in whorl 2, as in ovu mutants, the upper two organs of this whorl are also vestigial or aborted. The two upper organs are therefore aborted whether stamens grow in whorls 2 or 3 . This suggests that $c y c$ interacts with primordia in a similar way, irrespective of the whorl they occupy, and therefore that the fate of a primordium depends on a combinatorial interaction between functions determining whorl identity and the functions determining the differences between upper and lower organs. These observations have suggested a polar-coordinate model for the control of primordium fate (Fig. 8). The action of the whorl identity functions varies along the radius ( $r$ ) of the flower. The $c y c$ function varies along the vertical $(y)$ axis of the flower with its effect generally increasing from the lower to the upper parts of the axis. This results in a zygomorphic phenotype because for half of the flower, every organ has a unique specification, identical with its 'mirror image' in the other half (Fig. 8).

A further feature of the proposed model is that it can explain some unexpected effects of whorl identity mutations. For example, the uppermost carpel in the

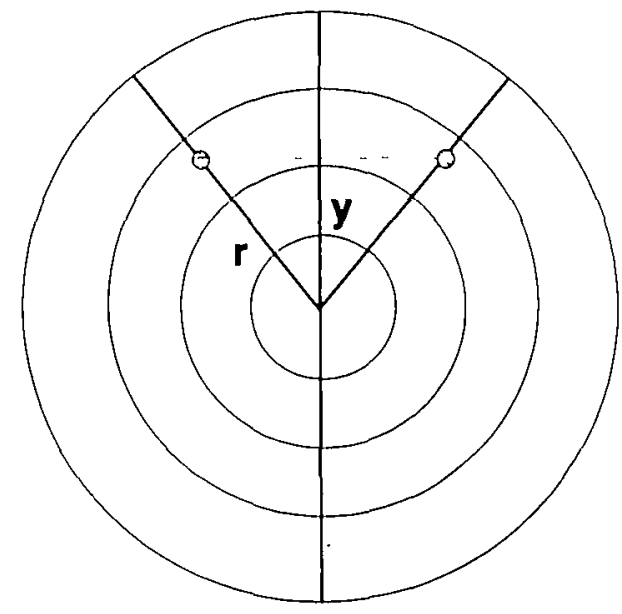

Fig. 8. Polar coordinate model for zygomorphy. The four whorls of the flower are shown as concentric rings. Expression of whorl identity functions varies along the radius (r) and the cyc function varies along the vertical (y) axis. Two primordia with the same combination of functions and hence the same developmental fate, are shown joined by a dotted horizontal line. Mutations eliminating the whorl identity functions result in some primordia in different whorls having similar specifications so that they adopt similar developmental fates. Mutations that abolish $c y c$ function remove differential expression along the y-axis such that all primordia in a whorl adopt a fate similar to that of the lower primordia of the wild-type whorl.

first whorl of ovu mutants is sterile and devoid of ovules, whereas the other carpels of this whorl contain ovules. This might be because the uppermost carpel is growing in a region of high cyc activity (i.e. at the top of the y-axis) not normally experienced by the wild-type carpels in whorl 4. Similarly, petals in the inner region of pleni, mutants often appear to be almost radially symmetrical because in more central whorls there is less difference in $c y c$ activity between upper and lower organs than in outer whorls.

\section{Terminal symmetry and inflorescence evolution}

Many species with zygomorphic flowers have indeterminate inflorescences that do not normally terminate in a flower (e.g. Antirrhinum, see earlier). However, exceptional plants which produce terminal flowers have been described in some of these species and in all cases the terminal flowers have radial symmetry (Peyritsch, 1870,1872 ). The inheritance of this phenotype has been studied in Antirrhinum and shown to be caused by a single recessive allele at a locus called centroradialis. The terminal flowers have petals resembling the lowest petal of wild type, similar to the flowers seen in $c y c$ mutations. However, the number of organs can be greater than 5, and we have observed large terminal flowers with as many as 14 sepals 12 petals and 11 stamens.

One explanation for the symmetrical terminal flower is based on the polar-coordinate model for zygomorphy described above. Flower meristems in axillary positions are in an asymmetrical environment with the main apex 
above them and a subtending bract below them. This could produce differences along the $y$-axis of the fioral meristem and these may provide the basis for establishing the gradient of cyc activity and hence zygomorphy. Terminal flowers would be in a radially symmetrical environment and therefore unable to establish a y-axis and activate $c y c$. The greater number of organs in terminal symmetric flowers might simply reflect the larger size of the main apex compared to axillary meristems.

Mutations such as centroradialis also have some important implications for inflorescence evolution. Some plants (e.g. Magnolia) have large single flowers at the termini of shoots (Fig. 9). In most species, however, flowers are clustered together in inflorescences which have been classified into two basic types: determinate and indeterminate (Weberling, 1989). In plants with determinate inflorescences, the apical inflorescence meristem is converted to a floral meristem and further floral meristems are produced in axillary positions or at the apex of side-branches (see example in Fig. 9). The main apex of indeterminate inflorescences is not converted into a floral meristem and flowers only arise in axillary positions (e.g. Antirrhinum).

Various evolutionary schemes have been proposed for the derivation of the various inflorescence types from a primitive condition (Parkin, 1914; Stebbins, 1974). One important feature of this evolutionary process is the change in timing and position at which inflorescence meristems switch to the floral pathway. The isolation of genes such as $f l o$, that regulate this transition, now opens these problems to molecular analysis. For example, in plants with single terminal flowers there may be no distinct inflorescence meristem and genes such as flo may be activated in the apex directly after vegetative growth. In determinate inflorescences, flo may be activated in the main apex and in the apices of side-branches. In indeterminate inflorescences, flo expression may be restricted to bracts and axillary meristems. The study of how flo is regulated in different plant species and also in mutants such as centroradialis, that convert indeterminate inflorescences to a determinate condition, should provide considerable new insights into inflorescence evolution.

\section{References}

Awasthi, D. K., Kumar, V. and Murty, Y. S. (1984). Flower development in Antirrhinum majus L. (Scrophulariaceae) with a comment upon corolla tube formation. Bot. Mag. Tokyo 97, 13-22.

Bowman, J. L., Smyth, D. R. ANd Meyerowitz, E. M. (1989). Genes directıng flower development in Arabidopsis. Plant Cell 1, 37-52.

Carpenter, R. and Coen, E. S. (1990). Floral homeotic mutations produced by transposon-mutagenesis in Antirrhinum majus Genes Dev. 4, 1483-1493.

Carpenter, R., Martin, C. R. and Coen, E. S. (1987).
Comparison of genetic behaviour of the transposable element Tam3 at two unlinked pigment loci in Antirrhinum majus. Molec. gen. Genet. 207, 82-89.

ChITTEnden, R. J. (1928). Note on an abnormal Antirrhinum. J. Genet. 19, 281-283

Coen, E. S., Robrins, T. P., Almeida, J., Hudson, A. and Carpenter, R. (1989). Consequences and mechanisms of transposition in Antirrhunum majus. In Moblle DNA (ed. D. E. Berg and M. M. Howe), pp. 413-436. American Society for Microbiology, Washıngton, DC.

Coen, E. S., Romero, J. M., Doyle, S., Elliott, R., Murphy, G AND CARPENTER, R. (1990). floricaula: a homeotic gene required for flower development in Antirrhinum majus. Cell 63, 1311-1322.

Harrison, B. J. AND FinCham, J. R. S. (1964). Instability at the Pal locus in Anturrhinum majus. 1. Effects of environment on frequencies of somatic and germinal mutation. Heredity 19 , 237-258.

Haughn, G. W. and Sommeville, C R. (1988). Genetic control of morphogenesis in Arabidopsis. Devl Genet. 9, 73-89.

Heslop-Harrison, J. (1964). Sex expression in flowering plants. Brookhaven Symp. Biol. 16, 109-125.

Imal, Y. (1934). On the mutable genes of Pharbins, with special reference to their bearing on the mechanism of bud-variation. $J$. Coll. Agric. 12, 479-523

InGHaM, P. W. (1988). The molecular genetics of embryonic pattern formation in Drosophila. Nature 335, 25-34.

KinG, R. C. (1974). Handbook of Genellcs, vol. 2. New York: Plenum.

Kuckuck, H. ANd Schick, R. (1930). Die Erbfaktoren bel Antirrhinum majus und ihre Bezeichung. Z. indukt. Abstamm. u. VererbLehre. 56, 51-83.

LATCHMAN, D. S. (1990). Eukaryotic transcription factors. Biochem. J. 270, 281-289.

Mermod, N., O'Neill, E. A., Kelly, T. J. and Tulan, R. (1989). The proline rich transcriptional activator of CTF/NF-1 is distinct from the replication and DNA bındıng domain Cell $\mathbf{5 8}$. $741-753$.

Parkin, J. (1914). The evolution of the inflorescence. Limn. J. Bot. 42, 511-604.

Peyritsch, J. (1870). Uber Pelorien bei Labiaten. Sber. Akad. Wiss. Wien 62, 1-27.

Peyritsch, J. (1872). Uber Pelorienbildungen. Sber. Akad. Wiss. Wien 66, 1-35.

Schwarz-Sommer, Z., Huuser, P., Nacken, W., Saedler, H. and SOMmER, H. (1990). Genetic control of flower development: homeotic genes in Antirrhinum majus Science 250, 931-936.

Sommer, H., Beltrán, J., Huuser, P., Pape, H., Lonnig, W., SaEdler, H. ANd Schwarz-Sommer, Z. (1990). Deficiens, a homeotic gene involved in the control of flower morphogenesis in Anturrhinum majus: the protein shows homology to tanscription factors. EMBO J. 9, 605-613.

Stebins, G. L. (1974). Flowering Plants. Evolution Above the Species Level. Harvard University Press, Cambridge, Massachusetts

Stubre, H. (1966). Genetik und Zytologıe von Antirrhınum L sect. Antirrhinum. Veb Gustav Fischer Verlag, Jena.

Struhl, K. (1989). Helix-turn-helix, zinc-finger, and leucine-zipper motıfs for eukaryotic transcriptional regulatory proteins. Trends biochem. Scl. 14, 137-140.

Tilney-Basset, R. A. E. (1986). Plant Chimeras. E. Arnold, London.

Weberling, F. (1989). Morphology of Flowers and Inflorescences Cambridge University Press, Cambridge.

YANOFSKY, M. F., Ma, H., Bowman, J. L., Drews, G. N., Feldman, K. A. And Meyerowitz, E M. (1990). AGAMOUS: an Arabidopsis homeotic gene whose product resembles transcription factors. Nature 346, 35-39. 